\title{
Nonlinear Super Integrable Couplings of Super Classical Boussinesq Hierarchy
}

\author{
Sixing $\mathrm{Tao}^{1 *}$, Hui Shi ${ }^{2}$ \\ 1 School of Mathematics and Information Science, Shangqiu Normal University, Shangqiu 476000, China. \\ 2 School of Physics and Electronic Information, Shangqiu Normal University, Shangqiu, 476000, China. \\ * Corresponding author. Tel.:+8613781680641; email: taosixing@163.com \\ Manuscript submitted January 10, 2015; accepted June 5, 2015. \\ doi: 10.17706/ijapm.2015.5.3.177-184
}

\begin{abstract}
Enlargement of Lie super algebra $B(0,1)$ was given firstly. Then nonlinear super integrable couplings of the super classical Boussinesq hierarchy based upon this enlarged matrix Lie super algebra were constructed secondly. And its super Hamiltonian structures were established by using super trace identity thirdly. As its reduction, special integrable couplings of classical Boussinesq hierarchy were obtained finally.
\end{abstract}

Key words: Lie super algebra, super integrable couplings,super classical Boussinesq hierarchy, super Hamiltonian structures.

\section{Introduction}

With the development of soliton theory, super integrable systems associated with Lie super algebra have aroused growing attentions by many mathematicians and physicists. It was known that super integrable systems contained the odd variables, which would provide more prolific fields for mathematical researchers and physical ones. Several super integrable systems including super AKNS hierarchy, super KdV hierarchyand super classical Boussinseq hierarchy, etc., have been studied [1]-[4]. There are some interesting results on the super integrable systems, such as Darboux transformation [5], super Hamiltonian structures [6], binary nonlinearization [7] and reciprocal transformation [8] and so on.

The research of integrable couplings of the well knownintegrable hierarchy has been received considerable attentions [9]-[12]. One approach to construct linear integrable couplings of the classical soliton equation are presented by using matrix Lie algebra constructing new loop Lie algebra [13]. Recently, Ma and Zhu [14], [15] presented a scheme for constructing nonlinear continuous and discrete integrable couplings using the block type matrix algebra. However, there is one interesting question for us is how to generate nonlinear super integrable couplings for the super integrable hierarchy.

In this paper, We take the Lie algebra $B(0,1)$ as an example to illustrate the approach for extending Lie super algebras. Based on the enlarged Lie super algebra $g l(6,2)$, we work out nonlinear super integrable Hamiltonian couplings of the super classical Boussinesq hierarchy. Finally, we will reduce the nonlinear super super classical Boussinesqintegrable Hamiltonian couplings to some special cases.

\section{Enlargement of Lie Super Algebra}

Consider the Lie super algebra $B(0,1)$. Its basis is 


$$
E_{1}=\left(\begin{array}{ccc}
1 & 0 & 0 \\
0 & -1 & 0 \\
0 & 0 & 0
\end{array}\right), E_{2}=\left(\begin{array}{lll}
0 & 1 & 0 \\
0 & 0 & 0 \\
0 & 0 & 0
\end{array}\right), E_{3}=\left(\begin{array}{lll}
0 & 0 & 0 \\
1 & 0 & 0 \\
0 & 0 & 0
\end{array}\right), E_{4}=\left(\begin{array}{ccc}
0 & 0 & 1 \\
0 & 0 & 0 \\
0 & -1 & 0
\end{array}\right), E_{5}=\left(\begin{array}{lll}
0 & 0 & 0 \\
0 & 0 & 1 \\
1 & 0 & 0
\end{array}\right) .
$$

where $E_{1}, E_{2}, E_{3}$ are even element and $E_{4}, E_{5}$ are odd elements. Their non-zero (anti) commutation relations are

$$
\begin{aligned}
& {\left[E_{1}, E_{2}\right]=2 E_{2},\left[E_{1}, E_{3}\right]=-2 E_{3},\left[E_{1}, E_{4}\right]=E_{4},\left[E_{1}, E_{5}\right]=-E_{5},\left[E_{2}, E_{3}\right]=E_{1},\left[E_{2}, E_{5}\right]=E_{4},} \\
& {\left[E_{3}, E_{4}\right]=E_{5},\left[E_{4}, E_{4}\right]=-2 E_{2},\left[E_{4}, E_{5}\right]=E_{1},\left[E_{5}, E_{5}\right]=2 E_{3} .}
\end{aligned}
$$

Let us enlarge the Lie super algebra $B(0,1)$ to the Lie super algebra $g l(6,2)$ with a basis

$$
\begin{aligned}
& e_{1}=\left(\begin{array}{ccccc}
1 & 0 & 0 & 0 & 0 \\
0 & -1 & 0 & 0 & 0 \\
0 & 0 & 1 & 0 & 0 \\
0 & 0 & 0 & -1 & 0 \\
0 & 0 & 0 & 0 & 0
\end{array}\right), e_{2}=\left(\begin{array}{lllll}
0 & 1 & 0 & 0 & 0 \\
0 & 0 & 0 & 0 & 0 \\
0 & 0 & 0 & 1 & 0 \\
0 & 0 & 0 & 0 & 0 \\
0 & 0 & 0 & 0 & 0
\end{array}\right), e_{3}=\left(\begin{array}{lllll}
0 & 0 & 0 & 0 & 0 \\
1 & 0 & 0 & 0 & 0 \\
0 & 0 & 0 & 0 & 0 \\
0 & 0 & 1 & 0 & 0 \\
0 & 0 & 0 & 0 & 0
\end{array}\right), e_{4}=\left(\begin{array}{ccccc}
0 & 0 & 1 & 0 & 0 \\
0 & 0 & 0 & -1 & 0 \\
0 & 0 & 1 & 0 & 0 \\
0 & 0 & 0 & -1 & 0 \\
0 & 0 & 0 & 0 & 0
\end{array}\right), \\
& e_{5}=\left(\begin{array}{lllll}
0 & 0 & 0 & 1 & 0 \\
0 & 0 & 0 & 0 & 0 \\
0 & 0 & 0 & 1 & 0 \\
0 & 0 & 0 & 0 & 0 \\
0 & 0 & 0 & 0 & 0
\end{array}\right), e_{6}=\left(\begin{array}{lllll}
0 & 0 & 0 & 0 & 0 \\
0 & 0 & 1 & 0 & 0 \\
0 & 0 & 0 & 0 & 0 \\
0 & 0 & 1 & 0 & 0 \\
0 & 0 & 0 & 0 & 0
\end{array}\right), e_{7}=\left(\begin{array}{ccccc}
0 & 0 & 0 & 0 & 1 \\
0 & 0 & 0 & 0 & 0 \\
0 & 0 & 0 & 0 & 0 \\
0 & 0 & 0 & 0 & 0 \\
0 & -1 & 0 & 1 & 0
\end{array}\right), e_{8}=\left(\begin{array}{ccccc}
0 & 0 & 0 & 0 & 0 \\
0 & 0 & 0 & 0 & 1 \\
0 & 0 & 0 & 0 & 0 \\
0 & 0 & 0 & 0 & 0 \\
1 & 0 & -1 & 0 & 0
\end{array}\right) .
\end{aligned}
$$

where $e_{1}, e_{2}, e_{3}, e_{4}, e_{5}, e_{6}$ are even, and $e_{7}, e_{8}$ are odd.

The generator of Lie super algebra $g l(6,2), e_{i}(1 \leq i \leq 8)$ satisfy the following (anti) commutation relations:

$\left[e_{1}, e_{2}\right]=2 e_{2},\left[e_{1}, e_{3}\right]=-2 e_{3},\left[e_{1}, e_{5}\right]=2 e_{5},\left[e_{1}, e_{6}\right]=-2 e_{6},\left[e_{1}, e_{7}\right]=e_{7},\left[e_{1}, e_{8}\right]=-e_{8},\left[e_{2}, e_{3}\right]=e_{1}$,

$\left[e_{2}, e_{4}\right]=-2 e_{5},\left[e_{2}, e_{6}\right]=e_{4},\left[e_{2}, e_{8}\right]=e_{7},\left[e_{3}, e_{4}\right]=2 e_{6},\left[e_{3}, e_{5}\right]=-e_{4},\left[e_{3}, e_{7}\right]=e_{8},\left[e_{4}, e_{5}\right]=2 e_{5}$,

$\left[e_{4}, e_{6}\right]=-2 e_{6},\left[e_{5}, e_{6}\right]=e_{4},\left[e_{7}, e_{7}\right]=2 e_{5}-2 e_{2},\left[e_{7}, e_{8}\right]=e_{1}-e_{4},\left[e_{8}, e_{8}\right]=2 e_{3}-2 e_{6}$,

$\left[e_{1}, e_{4}\right]=\left[e_{2}, e_{5}\right]=\left[e_{2}, e_{7}\right]=\left[e_{3}, e_{6}\right]=\left[e_{3}, e_{8}\right]=\left[e_{4}, e_{7}\right]=\left[e_{4}, e_{8}\right]=\left[e_{5}, e_{7}\right]=\left[e_{5}, e_{8}\right]=\left[e_{6}, e_{7}\right]=\left[e_{6}, e_{8}\right]=0$.

Define a loop super algebra corresponding to the Lie super algebra $g l(6,2)$, denote by

$$
g l(6,2) \otimes C\left[\lambda, \lambda^{-1}\right]=\left\{e_{i} \lambda^{m} \mid e_{i} \in g l(6,2), i=1, \cdots, 8 ; m=0, \pm 1, \pm 2, \cdots\right\} .
$$

The corresponding (anti)commutative relations are given as

$$
\left[e_{i} \lambda^{m}, e_{j} \lambda^{n}\right]=\left[e_{i}, e_{j}\right] \lambda^{m+n}, \forall e_{i}, e_{j} \in g l(6,2) .
$$

\section{Nonlinear Super Integrable couplings of the Super Classical Boussinesq Hierarchy}

If let us start from an enlarged spectral problem associated with $g l(6,2)$,

$$
\phi_{x}=U(u, \lambda) \phi, U=-e_{1}(1)-\frac{1}{4} q e_{1}(0)+r e_{2}(0)-e_{3}(0)+u_{1} e_{4}(0)+u_{2} e_{5}(0)+\alpha e_{7}(0)+\beta e_{8}(0)
$$




$$
=\left(\begin{array}{ccccc}
-\frac{1}{2} \lambda+\frac{1}{2} q & -r & u_{1} & u_{2} & \alpha \\
1 & \frac{1}{2} \lambda-\frac{1}{2} q & 0 & -u_{1} & \beta \\
0 & 0 & -\frac{1}{2} \lambda+\frac{1}{2} q+u_{1} & -r+u_{2} & 0 \\
0 & 0 & 1 & \frac{1}{2} \lambda-\frac{1}{2} q-u_{1} & 0 \\
\beta & -\alpha & -\beta & \alpha & 0
\end{array}\right) .
$$

where $q, r, u_{1}, u_{2}$ are even potentials, but $\alpha, \beta$ are odd ones.

In order to obtain super integrable couplings of super integrable hierarchy, we first solve the adjoint representation of (7),

$$
V_{x}=[U, V],
$$

with

$$
V=A e_{1}(0)+B e_{2}(0)+C e_{3}(0)+E e_{4}(0)+F e_{5}(0)+G e_{6}(0)+\rho e_{7}(0)+\delta e_{8}(0)=\left(\begin{array}{ccccc}
A & B & E & F & \rho \\
C & -A & G & -E & \delta \\
0 & 0 & A+E & B+F & 0 \\
0 & 0 & C+G & -A-E & 0 \\
\delta & -\rho & -\delta & \rho & 0
\end{array}\right) .
$$

where $A, B, C, E, F$ and $G$ are commuting fields, and $\rho, \delta$ are anti-commuting fields.

Substituting

$$
A=\sum_{m \geq 0} A_{m} \lambda^{-m}, B=\sum_{m \geq 0} B_{m} \lambda^{-m}, C=\sum_{m \geq 0} C_{m} \lambda^{-m}, E=\sum_{m \geq 0} E_{m} \lambda^{-m}, F=\sum_{m \geq 0} F_{m} \lambda^{-m}, G=\sum_{m \geq 0} G_{m} \lambda^{-m}, \rho=\sum_{m \geq 0} \rho_{m} \lambda^{-m}, \delta=\sum_{m \geq 0} \delta_{m} \lambda^{-m} .
$$

Intoprevious equation gives the following recursive formulas

$$
\left\{\begin{array}{l}
A_{m, x}=B_{m}+r C_{m}+\beta \rho_{m}+\alpha \delta_{m}, \\
B_{m, x}=-2 r A_{m}-2 B_{m+1}-\frac{1}{2} q B_{m}-2 \alpha \rho_{m}, \\
C_{m, x}=-2 A_{m}+2 C_{m+1}+\frac{1}{2} q C_{m}+2 \beta \delta_{m}, \\
E_{m, x}=u_{2} C_{m}+F_{m}+r G_{m}+u_{2} G_{m}-\beta \rho_{m}-\alpha \delta_{m}, \\
F_{m, x}=-2 u_{2} A_{m}+2 u_{1} B_{m}-2 r E_{m}-2 u_{2} E_{m}-2 F_{m+1}-\frac{1}{2} q F_{m}+2 u_{1} F_{m}+2 \alpha \rho_{m}, \\
G_{m, x}=-2 u_{1} C_{m}-2 E_{m}+2 G_{m+1}+\frac{1}{2} q G_{m}-2 u_{1} G_{m}-2 \beta \delta_{m}, \\
\rho_{m, x}=-\alpha A_{m}-\beta B_{m}-\rho_{m+1}-\frac{1}{4} q \rho_{m}+r \delta_{m}, \\
\delta_{m, x}=\beta A_{m}-\alpha C_{m}+\rho_{m}+\delta_{m+1}+\frac{1}{4} q \delta_{m} .
\end{array}\right.
$$

From previous equations, we can successively deduce

$$
\begin{aligned}
& A_{0}=1, B_{0}=C_{0}=F_{0}=G_{0}=\rho_{0}=\delta_{0}=0, E_{0}=\varepsilon=\text { const., } A_{1}=0, B_{1}=-r, C_{1}=1, E_{1}=0, F_{1}=-u_{2}-\varepsilon r-\varepsilon u_{2}, G_{1}=\varepsilon, \\
& \rho_{1}=-\alpha, \delta_{1}=-\beta, A_{2}=\frac{1}{2} r-\alpha \beta, B_{2}=\frac{1}{2} r_{x}+\frac{1}{4} q r, C_{2}=-\frac{1}{4} q, E_{2}=\frac{1}{2} u_{2}+\frac{1}{2} \varepsilon u_{2}+\frac{1}{2} \varepsilon r+\alpha \beta, F_{2}=\frac{1}{2} u_{2 x}+\frac{1}{2} \varepsilon u_{2 x}+\frac{1}{2} \varepsilon r_{x}-r u_{1} \\
& +\frac{1}{4} \varepsilon q r+\frac{1}{4} q u_{2}+\frac{1}{4} \varepsilon q u_{2}-u_{1} u_{2}-\varepsilon u_{1} u_{2}-\varepsilon r u_{1}, G_{2}=u_{1}+\varepsilon u_{1}-\frac{1}{4} \varepsilon q, \rho_{2}=\alpha_{x}+\frac{1}{4} q \alpha, \delta_{2}=-\beta_{x}+\frac{1}{4} q \beta, A_{3}=-\frac{1}{4} r_{x}+ \\
& -\frac{1}{4} q r+\alpha_{x} \beta-\alpha \beta_{x}+\frac{1}{2} q \alpha \beta, B_{3}=-\frac{1}{4} r_{x x}-\frac{1}{8} q_{x} r-\frac{1}{4} q r_{x}-\frac{1}{2} r^{2}-\frac{1}{16} q^{2} r+r \alpha \beta-\alpha \alpha_{x}, C_{3}=-\frac{1}{8} q_{x}+\frac{1}{2} r+\frac{1}{16} q^{2}-\alpha \beta+\beta \beta_{x}, \\
& E_{3}=-\frac{1}{4} q u_{2}-\frac{1}{4} \varepsilon q u_{2}-\frac{1}{4} \varepsilon q r+r u_{1}+\varepsilon r u_{1}-\frac{1}{4} \varepsilon r_{x}+u_{1} u_{2}+\varepsilon u_{1} u_{2}-\frac{1}{4} u_{2 x}-\frac{1}{4} \varepsilon u_{2 x}-\alpha_{x} \beta+\alpha \beta_{x}-\frac{1}{2} q \alpha \beta, F_{3}=(\varepsilon+1) . \\
& \left(-r u_{1}^{2}-u_{1}^{2} u_{2}-\frac{1}{8} q_{x} u_{2}-\frac{1}{4} q u_{2 x}+\frac{1}{2} u_{1 x} u_{2}+u_{1} u_{2 x}+r_{x} u_{1}-\frac{1}{2} r u_{1 x}-\frac{1}{2} u_{2}^{2}-\frac{1}{4} u_{2 x x}-\frac{1}{16} q^{2} u_{2}+\frac{1}{2} q r u_{1}+\frac{1}{2} q u_{1} u_{2}-r u_{2}\right)-r \alpha \beta \\
& +\alpha \alpha_{x}-\frac{1}{16} \varepsilon q^{2} r-\frac{1}{4} \varepsilon q r_{x}-\frac{1}{8} \varepsilon q_{x} r-\frac{1}{2} \varepsilon r^{2}-\frac{1}{4} \varepsilon r_{x x}, G_{3}=\frac{1}{2} u_{1 x}+\frac{1}{2} \varepsilon u_{1 x}-\frac{1}{8} \varepsilon q_{x}+\frac{1}{2} u_{2}+\frac{1}{2} \varepsilon u_{2}-2 \varepsilon r+\alpha \beta-\frac{1}{2} q u_{1}-\frac{1}{2} \varepsilon q u_{1}+\frac{1}{16} \varepsilon q^{2} \\
& +u_{1}^{2}+\varepsilon u_{1}^{2}-\beta \beta_{x}, \rho_{3}=-\alpha_{x x}-\frac{1}{4} q_{x} \alpha-\frac{1}{2} q \alpha_{x}-\frac{1}{2} r \alpha-\frac{1}{2} r_{x} \beta-\frac{1}{16} q^{2} \alpha-4 r \beta_{x}, \delta_{3}=-\beta_{x x}+\frac{1}{4} q_{x} \beta+\frac{1}{2} q \beta_{x}-\frac{1}{2} r \beta+\alpha_{x}-\frac{1}{16} q^{2} \beta .
\end{aligned}
$$

Equations (11) can be written as 


$$
\left(\begin{array}{c}
-A_{m+1}-\frac{1}{2} E_{m+1} \\
2 C_{m+1}+G_{m+1} \\
2 A_{m+1}+2 E_{m+1} \\
C_{m+1}+G_{m+1} \\
2 \delta_{m+1} \\
-2 \rho_{m+1}
\end{array}\right)=L\left(\begin{array}{c}
-A_{m}-\frac{1}{2} E_{m} \\
2 C_{m}+G_{m} \\
2 A_{m}+2 E_{m} \\
C_{m}+G_{m} \\
2 \delta_{m} \\
-2 \rho_{m}
\end{array}\right)
$$

where

$$
L=\left(\begin{array}{cccccc}
-\frac{1}{2} \partial-\frac{1}{4} \partial^{-1} q \partial & -\frac{1}{4} r-\frac{1}{4} \partial^{-1} r \partial & -\frac{1}{4} \partial^{-1} u_{1} \partial & -\frac{1}{4} u_{2}-\frac{1}{4} \partial^{-1} u_{2} \partial & -\frac{1}{8} \alpha-\frac{1}{4} \partial^{-1} \alpha \partial & \frac{1}{8} \beta-\frac{1}{4} \partial^{-1} \beta \partial \\
-2 & \frac{1}{2} \partial-\frac{1}{4} q & 0 & u_{1} & -\frac{1}{2} \beta & 0 \\
0 & 0 & -\frac{1}{2} \partial-\frac{1}{4} \partial^{-1} q \partial+\partial^{-1} u_{1} \partial & r+u_{2}+\partial^{-1} r \partial+\partial^{-1} u_{2} \partial & 0 & 0 \\
0 & 0 & \frac{1}{2} & \frac{1}{2} \partial-2 u_{1}-\frac{1}{4} q & 0 & 0 \\
4 \beta & 2 \alpha & \beta & -2 \alpha & \partial-\frac{1}{4} q & -1 \\
-4 \alpha-4 \beta \partial & -2 r \beta & -\beta \partial & 2 r \beta & -r+\alpha \beta & -\partial-\frac{1}{4} q
\end{array}\right) .
$$

Then, let us consider the spectral problem (7) with the following auxiliary problem

$$
\phi_{t_{n}}=V^{(n)} \phi
$$

with

$$
V^{(n)}=\sum_{j=0}^{n}\left(\begin{array}{ccccc}
A_{j} & B_{j} & E_{j} & F_{j} & \rho_{j} \\
C_{j} & -A_{j} & G_{j} & -E_{j} & \delta_{j} \\
0 & 0 & A_{j}+E_{j} & B_{j}+F_{j} & 0 \\
0 & 0 & C_{j}+G_{j} & -A_{j}-E_{j} & 0 \\
\delta_{j} & -\rho_{j} & -\delta_{j} & \rho_{j} & 0
\end{array}\right) \lambda^{n-j}-C_{n+1} e_{1}(0)-G_{n+1} e_{4}(0),
$$

From the compatible condition $\phi_{x, t_{n}}=\phi_{t_{n}, x}$, according to (7) and (14), we get the zero curvature equation

$$
U_{t_{n}}-V_{x}^{(n)}+\left[U, V^{(n)}\right]=0
$$

which gives a nonlinear Lax super integrable hierarchy

$$
u_{t_{n}}=\left(\begin{array}{c}
q \\
r \\
u_{1} \\
u_{2} \\
\alpha \\
\beta
\end{array}\right)_{t_{n}}\left(\begin{array}{c}
4 C_{n+1, x} \\
-2 B_{n+1}-2 r C_{n+1} \\
-G_{n+1, x} \\
-2 u_{2} C_{n+1}-2 F_{n+1}-2 r G_{n+1}-u_{2} G_{n+1} \\
-\alpha C_{n+1}-\rho_{n+1} \\
\beta C_{n+1}+\delta_{n+1}
\end{array}\right) \text {. }
$$

The super integrable hierarchy (17) is a nonlinear super integrablecouplings for the super classical Boussinesq hierarchy 


$$
\tilde{u}_{t_{n}}=\left(\begin{array}{c}
q \\
r \\
\alpha \\
\beta
\end{array}\right)_{t_{n}}=\left(\begin{array}{c}
4 C_{n+1, x} \\
-2 B_{n+1}-2 r C_{n+1} \\
-\alpha C_{n+1}-\rho_{n+1} \\
\beta C_{n+1}+\delta_{n+1}
\end{array}\right) \text {. }
$$

\section{Super Hamiltonian Structure}

A direct calculation reads

$$
\operatorname{Str}\left(U_{\lambda}, V\right)=-4 A-2 E, \operatorname{Str}\left(U_{q}, V\right)=-A-\frac{1}{2} E, \operatorname{Str}\left(U_{r}, V\right)=2 C+G, \operatorname{Str}\left(U_{u_{1}}, V\right)=2 A+2 E, \operatorname{Str}\left(U_{u_{2}}, V\right)=C+G, \operatorname{Str}\left(U_{\alpha}, V\right)=2 \delta, \operatorname{Str}\left(U_{\beta}, V\right)=-2 \rho .
$$

Substituting above results into the super trace identity [6]

$$
\frac{\delta}{\delta u} \int \operatorname{Str}\left(\frac{\delta U}{\delta \lambda} V\right) \mathrm{d} x=\lambda^{-\gamma} \frac{\partial}{\partial \lambda} \lambda^{\gamma} \operatorname{Str}\left(\frac{\delta U}{\delta u} V\right)
$$

yields that

$$
\frac{\delta}{\delta u} \int(-4 A-2 E) \mathrm{d} x=\lambda^{-\gamma} \frac{\partial}{\partial \lambda} \lambda^{\gamma}\left(\begin{array}{c}
-A+\frac{1}{2} E \\
2 C+G \\
2 A+2 E \\
C+G \\
2 \delta \\
-2 \rho
\end{array}\right)
$$

Comparing the coefficients of $\lambda^{-n-1}$ on both side of (21)

$$
\frac{\delta}{\delta u} \int\left(-4 A_{n+1}-2 E_{n+1}\right) \mathrm{d} x=\lambda^{-\gamma} \frac{\partial}{\partial \lambda} \lambda^{\gamma}\left(\begin{array}{c}
-A_{n}-\frac{1}{2} E_{n} \\
2 C_{n}+G_{n} \\
2 A_{n}+2 E_{n} \\
C_{n}+G_{n} \\
2 \delta_{n} \\
-2 \rho_{n}
\end{array}\right), n \geq 0 .
$$

From the initial values in (11), we obtain $\gamma=0$. Thus we have

$$
\frac{\delta H_{n}}{\delta u}=\left(\begin{array}{c}
-A_{n}-\frac{1}{2} E_{n} \\
2 C_{n}+G_{n} \\
2 A_{n}+2 E_{n} \\
C_{n}+G_{n} \\
2 \delta_{n} \\
-2 \rho_{n}
\end{array}\right), H_{n}=\int \frac{4 A_{n+1}+2 E_{n+1}}{n+1} \mathrm{~d} x, n \geq 0
$$

It then follows that the nonlinear super integrable couplings (17) possess the following super 
Hamiltonian form

$$
u_{t_{n}}=K_{n}(u)=J \frac{\delta H_{n}}{\delta u}
$$

where

$$
J=\left(\begin{array}{cccccc}
0 & 4 \partial & 0 & -4 \partial & 0 & 0 \\
4 \partial & 0 & \partial & 0 & \alpha & -\beta \\
0 & \partial & 0 & -2 \partial & 0 & 0 \\
-4 \partial & 0 & -2 \partial & 0 & -\alpha & \beta \\
0 & -\alpha & 0 & \alpha & 0 & \frac{1}{2} \\
0 & \beta & 0 & -\beta & \frac{1}{2} & 0
\end{array}\right) .
$$

is a super Hamiltonian operator and $H_{n}(n \geq 0)$ are Hamiltonian functions.

\section{Reductions}

Taking $\alpha=\beta=0$, the hierarchy (24) reduces to a nonlinear integrable couplings of the classical Boussinesqhirerarchy.

When $n=2$ in (24), we obtain the nonlinear super integrable couplings of the second order superclassical Boussinesqequatiaons

$$
\left\{\begin{array}{l}
q_{t_{2}}=-\frac{1}{2} q_{x x}+2 r_{x}+\frac{1}{2} q q_{x}-4 \alpha_{x} \beta-4 \alpha \beta_{x}+4 \beta \beta_{x x}, \\
r_{t_{2}}=\frac{1}{2} r_{x x}+\frac{1}{2} q_{x} r+\frac{1}{2} q r_{x}+2 \alpha \alpha_{x}-2 r \beta \beta_{x}, \\
u_{1, t_{2}}=(\varepsilon+1)\left(-\frac{1}{2} u_{1 x x}+\frac{1}{2} q_{x} u_{1}+\frac{1}{2} q u_{1 x}-2 u_{1} u_{1 x}-\frac{1}{2} u_{2 x}-\frac{1}{8} q_{x x}-\frac{1}{2} r_{x}-\frac{1}{8} q q_{x}\right)-\frac{1}{8} q_{x x}+\frac{1}{2} r_{x}+\frac{1}{8} q q_{x}-\alpha_{x} \beta-\alpha \beta_{x}+\beta \beta_{x x}, \\
u_{2, t_{2}}=(\varepsilon+1)\left(\frac{1}{2} q_{x} u_{2}-2 r u_{1 x}-2 u_{1 x} u_{2}-2 u_{1} u_{2 x}+\frac{1}{2} q u_{2 x}-2 r_{x} u_{1}+\frac{1}{2} u_{2 x x}+\frac{1}{2} q r_{x}+\frac{1}{2} q_{x} r+\frac{1}{2} r_{x x}\right)-\frac{1}{2} r_{x x}-\frac{1}{2} q_{x} r-\frac{1}{2} q r_{x}-2 \alpha \alpha_{x}+2 r \beta \beta_{x}, \\
\alpha_{t_{2}}=\alpha_{x x}+\frac{3}{8} q_{x} \alpha+\frac{1}{2} q \alpha_{x}+\frac{1}{2} r_{x} \beta+r \beta_{x}-\alpha \beta \beta_{x}, \\
\beta_{t_{2}}=-\beta_{x x}+\alpha_{x}+\frac{1}{8} q_{x} \beta+\frac{1}{2} q \beta_{x} .
\end{array}\right.
$$

Especially, taking $\alpha=\beta=0$ in (26), we can obtain the nonlinear integrable couplings of the second order classical Boussinesq equation

$$
\left\{\begin{array}{c}
q_{t_{2}}=-\frac{1}{2} q_{x x}+2 r_{x}+\frac{1}{2} q q_{x}, \\
r_{t_{2}}=\frac{1}{2} r_{x x}+\frac{1}{2} q_{x} r+\frac{1}{2} q r_{x}, \\
u_{1, t_{2}}=(\varepsilon+1)\left(-\frac{1}{2} u_{1 x x}+\frac{1}{2} q_{x} u_{1}+\frac{1}{2} q u_{1 x}-2 u_{1} u_{1 x}-\frac{1}{2} u_{2 x}-\frac{1}{8} q_{x x}-\frac{1}{2} r_{x}-\frac{1}{8} q q_{x}\right)-\frac{1}{8} q_{x x}+\frac{1}{2} r_{x}+\frac{1}{8} q q_{x}, \\
u_{2, t_{2}}=(\varepsilon+1)\left(\frac{1}{2} q_{x} u_{2}-2 r u_{1 x}-2 u_{1 x} u_{2}-2 u_{1} u_{2 x}+\frac{1}{2} q u_{2 x}-2 r_{x} u_{1}+\frac{1}{2} u_{2 x x}+\frac{1}{2} q r_{x}+\frac{1}{2} q_{x} r+\frac{1}{2} r_{x x}\right)-\frac{1}{2} r_{x x}-\frac{1}{2} q_{x} r-\frac{1}{2} q r_{x} .
\end{array}\right.
$$

If setting $\varepsilon=-1, u_{1}=\frac{1}{4} q, u_{2}=-r$ in (26), we obtain the second order super classical Boussinesq equation

$$
\left\{\begin{array}{l}
q_{t_{2}}=-\frac{1}{2} q_{x x}+2 r_{x}+\frac{1}{2} q q_{x}-4 \alpha_{x} \beta-4 \alpha \beta_{x}+4 \beta \beta_{x x}, \\
r_{t_{2}}=\frac{1}{2} r_{x x}+\frac{1}{2} q_{x} r+\frac{1}{2} q r_{x}+2 \alpha \alpha_{x}-2 r \beta \beta_{x}, \\
\alpha_{t_{2}}=\alpha_{x x}+\frac{3}{8} q_{x} \alpha+\frac{1}{2} q \alpha_{x}+\frac{1}{2} r_{x} \beta+r \beta_{x}-2 \alpha \beta \beta_{x}, \\
\beta_{t_{2}}=-\beta_{x x}+\alpha_{x}+\frac{1}{8} q_{x} \beta+\frac{1}{2} q \beta_{x} .
\end{array}\right.
$$




\section{Conclusions}

In this paper, we introduced an approach for constructing nonlinear integrable couplings of super integrable hierarchy. Zhang [16] once employed two kinds of explicit Lie algebra $F$ and $G$ to obtain the nonlinear integrable couplings of the GJ hierarchy and Yang hierarchy, respectively. It is easy to see that Lie algebra $F$ given in [16] is isomorphic to the Lie algebra span $\left\{e_{1}, e_{2}, e_{3}, e_{4}, e_{5}, e_{6}\right\}$ in $g l(6,2)$. So we can obtain nonlinear integrable couplings of super GJ and Yang hierarchy easily. The method in this paper can be applied to other super integrable systems for constructing their super integrable couplings.

\section{Acknowledgment}

This work was supported by the Natural Science Foundation of Henan Province (No.132300410202), the Science and Technology Key Research Foundation of the Education Department of Henan Province (No. 14A110010), the Youth Backbone Teacher Foundation of Shangqiu Normal University(No. 2013GGJS02).

\section{References}

[1] Li, Y. S., \& Zhang, L. N. (1988). A note on the super AKNS equations. Journal of Physics A: Mathematical and General, 21(7), 1549-1552.

[2] Tu, M. H., \& Shaw, J. C. (1999). Hamiltonian structures of generalized manin-radul super-KdV and constrained super KP hierarchies. Journal of Mathematical Physics, 40(6), 3021-3034.

[3] Tao, S. X., \& Xia, T. C. (2010). The super-classical-boussinesq hierarchy and its super Hamiltonian structure. Chinese Physics B, 19(7), 070202.

[4] Tao, S. X., Wang, H., \& Shi, H. (2011). Binary nonlinearization of the super classical-boussinesq hierarchy. Chinese Physics B, 20(7), 070201.

[5] Aratyn, H., Nissimov, E., \& Pacheva, S. (1999). Supersymmetric kadomtsev-petviashvili hierarchy: "Ghost" symmetry structure, reductions, and Darboux-Bäcklundsolutions. Journal of Mathematical Physics, 40(6), 2922-2932.

[6] Ma, W. X., He, J. S., \& Qin, Z. Y. (2008). A supertrace identity and its applications to super integrable systems. Journal of Mathematical Physics, 49(3), 033511.

[7] He, J. S., Yu, J., Cheng, Y., \& Ma, W. X. (2008). Binary nonlinearization of the super AKNS system. Modern Physics Letters B, 22(4), 275-288.

[8] Liu, Q. P., Popowicz, Z., \& Tian, K. (2010). Supersymmetric reciprocal transformation and its applications. Journal of Mathematical Physics, 51(9), 093511.

[9] Ma, W. X. (2003). Enlarging spectral problems to construct integrable couplings of soliton equations. Physics Letters A, 316(1-2), 72-76.

[10] Ma, W. X., Xu, X. X., \& Zhang, Y. F. (2006). Semidirect sums of Lie algebras and discrete integrable couplings. Journal of Mathematical Physics, 47(5), 053501.

[11] Ma, W. X., \& Chen, M. (2006). Hamiltonian and quasi-Hamiltonian structures associated with semi-direct sums of Liealgebras. Journal of Physics A: Mathematical and General, 39(34), 10787-10801.

[12] Zhang, Y. F., \& Fan, E. G. (2010). Coupling integrable couplings and bi-Hamiltonian structure associated with the Boiti-Pempinelli-Tu hierarchy. Journal of Mathematical Physics, 51(8), 083506.

[13] Zhang, Y. F., \& Zhang, H. Q. (2002). A direct method for integrable couplings of TD hierarchy. Journal of Mathematical Physics, 43(1), 466-472.

[14] Ma, W. X. (2011). Nonlinear continuous integrable Hamiltonian couplings. Applied Mathematics and Computation, 217(17), 7238-7244.

[15] Ma, W. X, \& Zhu, Z. N. (2010). Constructing nonlinear discrete integrable Hamiltonian couplings. Computers \& Mathematics with Applications, 60(9), 2601-2608. 


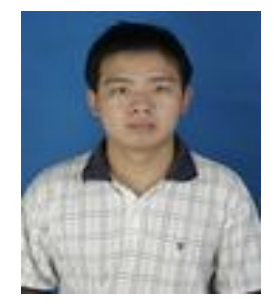

Sixing Tao was born in March 1981, in Qingzhou, Shandong Province of China. He received the bachelor degree in mathematics and applied mathematics from Qufu Normal University in 2003 and the master degree in basic mathematics from Qufu Normal University in 2006. He received the doctor degree in basic mathematics from Shanghai University in 2011. Now he is an associate professor in School of Mathematics and Information Science, Shangqiu Normal University. His research interests are in soliton theory and integrable systems. He has published more than 10 articles in recent years.

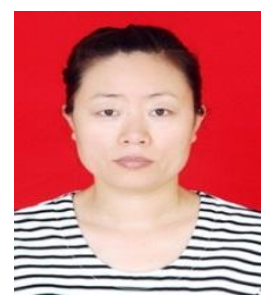

Hui Shi was born in September 1976, in Shangqiu, Henan province of China. She received the bachelor degree in automation from Henan Normal University in 2000 and the master degree in automation from Jiangsu University in 2008. Now she is a lecturer in School of Physics and Electronic Information, Shangqiu Normal University. Her research interests are in nonlinear control systems and applications. She has published more than 10 articles in recent years. 